\section{PM.12 THE MANAGEMENT OF PREGNANT WOMEN ATTENDING TRIAGE WITH SUSPECTED URINARY TRACT INFECTION (UTI)}

doi:10.1136/archdischild-2013-303966.097

\section{PM.14 HOW REASSURING IS A NORMALLY SITED PLACENTA AT THE 18 - 20+6 WEEK ANOMALY SCAN AT EXCLUDING PLACENTA PRAEVIA?}

doi:10.1136/archdischild-2013-303966.099

TE Mosedale, H Kither, L Byrd. St Mary's Hospital, Central Manchester University Hospitals NHS Trust, Manchester, UK

\begin{abstract}
Aims This audit and re-audit was undertaken to ascertain whether women presenting to Triage, with symptoms suggestive of possible UTI, are being appropriately assessed/treated.

Methods The Admission Proforma of all women attending Triage with lower abdominal pain with no clear cause, but where UTI was considered, was reviewed against agreed standards. Data was collected prospectively for a period of 2 and 3 weeks respectively. In each audit notes from 50 women were reviewed.

Results In the initial audit $86 \%$ (43/50) had urine dipped but only $5 \%(2 / 43)$ of these showed nitrites (1 had confirmed UTI); $32 \%$ $(16 / 50)$ were treated with antibiotics, $75 \%(12 / 16)$ of these had MSSU sent, but only 2 had confirmed UTI. In the re-audit $42 \%$ $(21 / 50)$ of patients had an MSSU - 75\% (6/8) of those prescribed antibiotics. Urine dipstick was performed in $94 \%(48 / 50)$ of all cases $-88 \%(7 / 8)$ of those treated. No nitrites were identified or UTI confirmed by culture.

Conclusions Pregnancy increases the risk for UTIs and failure to treat has serious maternal/neonatal consequences, not least preterm delivery. Nevertheless a diagnosis of UTI is too frequently made; often without strong evidence, and women are being overtreated. Antibiotic resistance is an increasing problem; unnecessary prescriptions must be avoided. All women presenting with abdominal pain should have their urine dipped. Audit confirms that leucocytes and/or proteinuria are not indicative of infection; even nitrites may not be diagnostic. If positive for nitrites, MSSU must be sent and urgent microscopy could be considered, ahead of prescribing antibiotics.
\end{abstract}

\section{PM.13 OFFSPRING BIRTH WEIGHT AND MATERNAL FASTING LIPIDS IN WOMEN SCREENED FOR GESTATIONAL DIABETES MELLITUS}

doi:10.1136/archdischild-2013-303966.098

V Dwyer, K Whyte, H Kelly, M Gibbs, A O'Higgins, MJ Turner. UCD Centre for Human Reproduction, Dublin, Ireland

Objective Maternal lipid metabolism is altered during pregnancy but little is known about the influence of these alterations on intrauterine fetal development. To examine the relationship between offspring birth weight and both fasting cholesterol and triglycerides in women screened selectively for Gestational Diabetes Mellitus (GDM).

Methods Prospective observational study in a large university maternity hospital. Women were recruited when they were screened selectively for GDM with a diagnostic $75 \mathrm{~g}$ Oral Glucose Tolerance Test (OGTT). At the time the fasting glucose was obtained an additional sample was taken for a lipid profile.

Results Of the 189 women recruited, the mean age was 32 years, $35.4 \%(n=67)$ were primigravidas, $44.1 \%(n=82)$ were obese and $11.6 \%(\mathrm{n}=22)$ had an abnormal OGTT. On univariate analysis, increased birth weight was correlated positively with multiparity, increased first trimester Body Mass Index (BMI), GDM and hypertriglyceridaemia but not cholesterol. On multivariate analysis, increased birth weight correlated positively only with hypertriglyceridaemia.

Conclusions This study provides further evidence that maternal hypertriglyceridaemia may be important in programming intrauterine fetal development and raises questions about whether women should be screened selectively for dyslipidaemia before, during and after pregnancy.
S Kemp, 0 Keag, G Tydeman. Victoria Hospital Kirkcaldy, NHS Fife, Kirckcaldy, UK

If the placenta abuts or overlies the os at the $18-20^{+6}$ week anomaly scan, management to identify those women who end up with placenta praevia (PP) is well established ${ }^{1}$. We could find no evidence of the reverse side of the issue: whether a normal placental site at the anomaly scan could be relied upon to exclude a diagnosis of PP later in pregnancy. Amongst clinicians in our region, there was widely differing opinion.

Between April 2003 and February 2012 there were approximately 32500 deliveries in NHS Fife $2.121(0.37 \%)$ women were identified who had been delivered by caesarean section where the indication was PP. The placental site from the anomaly scan was identified using the local ultrasound reporting system. Caldicott approval was granted.

There were 59 (49\%) emergency and 62 (51\%) elective caesarean sections. 111 (92\%) had an anomaly scan between 18 and $20^{+6}$ weeks

\begin{tabular}{lrll} 
Abstract PM.14 Table & & & \\
\hline Placental Site at 18 - 20+6 & $\mathbf{n}$ & & Later scan indicated' \\
\hline Not Low & 39 & $35 \%$ & No \\
Low but clear of os & 7 & $6 \%$ & No \\
Abutting os & 8 & $7 \%$ & Yes \\
Overlying os & 57 & $51 \%$ & Yes \\
Totals & 111 & & \\
\hline
\end{tabular}

In $46 / 111$ women $(41 \%, 95 \%$ CI $32-51 \%)$ the anomaly scan did not suggest the possibility of a problem. Hence, $3-5$ out of every 10 women with a normally sited placenta at their anomaly scan may ultimately develop PP. We believe that women who present later in pregnancy should have a further scan to exclude PP even if the placenta was not significantly low at the anomaly scan.

\section{REFERENCES}

1. Royal College of Obstetricians and Gynaecologists. Placenta praevia, placenta praevia accreta and vasa praevia: diagnosis and management. Green top guideline no. 27, January 2011

2. Information Services Division Scottish Morbidity Record; http://www.isdscotland org

\section{PM.15 NHS LOTHIAN GUIDELINES- ARE THEY BEING ADHERED TO FOR THE MANAGEMENT OF POSTPARTUM HAEMORRHAGE ( $>1.5$ L), PRE-ECLAMPSIA AND SEPSIS?}

doi:10.1136/archdischild-2013-303966.100

NU Sugiarto, KC Dundas, A Wise. The University of Edinburgh, Edinburgh, UK

Introduction Postpartum haemorrhage (PPH), pre-eclampsia and sepsis are the most common indications for receiving obstetrics high-dependency care in the Royal Infirmary of Edinburgh (RIE). This study therefore aimed to evaluate adherence to the NHS Lothian guidelines for the management of suspected severe sepsis (SSS), severe pre-eclampsia (SPE) and $\mathrm{PPH}>1.5 \mathrm{~L}$. Immediate resuscitation, ordering of investigations and treatment administration were evaluated.

Method A retrospective audit of paper and electronic patient records was performed on patients who received high dependency care for SSS, SPE and PPH > 1.5 L in the RIE.

Results 16 patients had SSS. All patients had IV access, their 'ABC' (Airway, Breathing, Circulation) checked, oxygen saturations, 